\title{
Joint internal moments in subjects with unilateral transtibial amputation during walking
}

\section{Francisco Molina-Rueda' ${ }^{1}$ Alicia Cuesta-Gómez ${ }^{1 *}$, María Carratalá-Tejada' ${ }^{1}$ Pilar Fernández-González' Juan Carlos Miangolarra-Page ${ }^{1,2}$, Isabel $M^{a}$ Alguacil-Diego'}

'Department of Physical Therapy, Occupational Therapy, Rehabilitation and Physical Medicine. Faculty of Health of Sciences. Rey Juan Carlos University, Madrid, Spain

${ }^{2}$ Physical Medicine and Rehabilitation Service of the University Hospital of Fuenlabrada, Madrid, Spain

\section{Article Info}

\section{Article Notes}

Received: October 05, 2020

Accepted: January 08, 2021

\section{*Correspondence:}

*Dr. Alicia Cuesta-Gómez, Department of Physical Therapy, Occupational Therapy, Rehabilitation and Physical Medicine. Faculty of Health of Sciences. Rey Juan Carlos University, Madrid, Spain; Telephone No: +34-914888948;

Email: alicia.cuesta@urjc.es.

-2021 Cuesta-Gómez A. This article is distributed under the terms of the Creative Commons Attribution 4.0 International License.

\section{Keywords}

Transtibial amputation

Gait

Joint kinetics

Joint internal moments

Three-dimensional gait analysis
Abstract

Background: Subjects with a unilateral transtibial amputation (UTA) that have used prosthesis for over five years have a higher occurrence rate of osteoarthritis at the knee and hip joints of the intact limb.

Objective: To research the joint internal moments of the hip, knee, and ankle in the sagittal and frontal planes during gait in persons with UTA.

Methods: 25 individuals with UTA (50.26 years \pm 13.76$)$ and 25 subjects without amputation (46.71 years \pm 13.76 ) participated in this study. Gait analysis was carried out using a Vicon ${ }^{\circledR}$ Motion System (Oxford Metrics, Oxford, UK) with eight $100 \mathrm{~Hz}$ cameras with infrared strobes, two $1000 \mathrm{~Hz} \mathrm{AMTI}{ }^{\circledR}$ forceplates.

Results: People with UTA walk with a greater hip extensor moment in both intact and prosthetic limbs. The hip abductor moment was lower on the prosthetic limb compared to the intact limb and the control group. At the knee joint, the subjects with UTA walked with a reduced knee extensor and valgus moment on their prosthetic limb compared to the control group. At the ankle joint, the statistical analysis showed that the individuals with UTA walked with a reduced plantarflexor moment during the stance period on the intact limb compared to the people without amputation.

Conclusions: Subjects with UTA walk with a different joint kinetic pattern in the sagittal and frontal planes compared to non-disabled individuals.

\section{Introduction}

The prevalence of limb amputation is expected to increase in the coming year ${ }^{1}$. Subjects with a unilateral transtibial amputation (UTA) that have used prosthesis for over five years have a higher occurrence rate of osteoarthritis (OA) at the knee and hip joints of the intact limb ${ }^{2}$. The literature has showed that the increased rate of OA occurs both for persons with vascular UTA and for subjects with trauma UTA. In this sense, modifications in walking pattern with alterations of ground reaction forces have been documented. The increased joint load exhibited by amputees with prosthesis may result in abnormal joint loading that, over time, may lead to joint pain and degeneration ${ }^{3,4}$.

Previous studies have only explained a small part of the adaptations that occur due to unilateral transtibial amputation during walking. The effects of unilateral transtibial amputation on the lower limb kinetics have not been sufficiently investigated. 
Beyaert et al. ${ }^{5}$ and Grumillier et al. ${ }^{6}$ showed that the hip and knee extensor moments were increased in the intact limb and decreased in the prosthetic limb compared to able bodied subjects. Recently, Orekhov et al. ${ }^{4}$ showed that the amputee knee extension moment was higher in the intact limb compared to the prosthetic limb during gait. In addition, Molina-Rueda et al. ${ }^{78}$ observed that the abductor hip moment was also increased in the intact limb and decreased in the prosthetic limb compared to controls. These alterations in joint moments could cause pain and an increased risk of osteoarthritis9.

The gait functions (progression, energy conservation and shock absorption) are possible by kinetic patterns that occur in the frontal and sagittal planes ${ }^{10}$. Therefore, a detailed examination of the joint moments carried out by the individuals with UTA during gait, is essential to produce new physical therapy guidelines and new approaches which could improve the quality of life of these people and reduce their disability.

The purpose of this study was to research the internal joint moments of the hip, knee, and ankle in the sagittal and frontal planes during gait in subjects with unilateral transtibial amputation.

\section{Methods}

\section{Participants}

Subjects with UTA were selected via consecutive non probabilistic sampling. The inclusion criteria for persons with amputation were as follows: regular users of the prosthesis, able to stand and walk independently without walking aids, and free from phantom limb pain. The exclusion criteria were presence of a known neurologic disorder, vestibular problems, marked visual deficiency, impaired cognitive function, and history of recent trauma, peripheral arterial disease affecting the lower limbs, fractures, and surgeries to the lower limb.

\section{Ethical aspects}

All participants provided a written informed consent prior to participation in this investigation, which was approved by the Ethics Committee at Rey Juan Carlos University (Madrid, Spain) and warranted its accordance with the Declaration of Helsinki.

\section{Procedure}

Gait analysis was carried out using the Vicon ${ }^{\circledR}$ Motion System (Oxford Metrics, Oxford, UK). This system is a three-dimensional motion analysis system consisting of eight $100 \mathrm{~Hz}$ cameras with infrared strobes, two $1000 \mathrm{~Hz}$ AMTI ${ }^{\circledR}$ force-plates (Watertown, USA), and a data station (Vicon MX control ${ }^{\circledR}$ ) where the information was gathered and processed ${ }^{7,8}$.
Special lightweight surface markers were attached directly to the skin or the prosthesis and placed over standardized landmarks on the intact side, prosthetic side and pelvis, or corresponding spots on the prosthesis (anterior and posterior superior iliac spines (left and right), lateral thigh, lateral femoral condyle, lateral shank, lateral malleoli, second metatarsal head, and posterior heel) according to the biomechanical model of the Vicon ${ }^{\circledR}$ Plug-in Gait ${ }^{11}$ as is mentioned in $^{7,8}$.

After the instrumentation, the individuals were instructed to walk along the 8-meter walkway while wearing their usual shoes (not athletic training shoes) and prosthesis. The participants were asked to walk at a selfselected comfortable gait speed.

\section{Data analysis}

We analyzed the peak of the lower limb internal moments during the stance period, with special regard to the hip extensor moment, the hip flexor moment, the hip abductor moment, the knee extensor moment, the knee flexor moment, the knee valgus moment and the ankle plantarflexor moment. We studied spatio-temporal parameters, such as walking velocity, cadence, and stride length.

The Vicon $®$ Nexus software v1.8.5 was used to calculate outcome measures based on the biomechanical model of the Vicon ${ }^{\circledR}$ Plug-in Gait ${ }^{11}$. Joint moment calculations were determined from synchronized coordinate and force data using an inverse dynamics approach ${ }^{12}$. Joint kinetics was normalized to body weight, and all parameters were normalized to $100 \%$ of the gait cycle. Internal moments were calculated and interpreted as the forces developed by the muscle and ligaments counteracting the moments produced by the ground reaction force ${ }^{13}$.

Five gait cycles of the prosthetic side, the intact side, and the control side were averaged for the data analysis. The foot contact events were defined automatically, using the "autocorrelation events" option of the Vicon $\AA$ Nexus software v1.8.5.

\section{Statistical analysis}

Statistical analysis was performed using SPSS 22.0. Shapiro and Wilk's W-statistic was used to screen all data for normality of distribution. The subjects were height/ weight matched. Single-factor analysis of variance (ANOVA) and Bonferroni's adjustment a posteriori tests were used to compare the sound side, prosthetic side, right control side, and left control side. Walking speed comparisons between the controls and persons with UTA were determined using Student's t-test. A significance level of 0.05 was used for all statistical comparisons.

\section{Sample size calculation}

To obtain a sample size estimation, we used the software 
G*Power 3.1.9.2. The effect size was estimated at 0.75 . The alpha error and the statistical power were set to 0.05 and 0.80 , respectively. According to these parameters, it was estimated that 23 subjects would be required for each group (subject with amputation vs. control group).

\section{Results}

The participants included 25 individuals with UTA (23 men, 2 women; 12 traumatic, 10 vascular, and 3 tumoral; 50.26 years $\pm 13.76 ; 80.02 \mathrm{~kg} \pm 13.79 ; 173.17 \mathrm{~cm} \pm 8.55)$ and 25 non-amputees as control ( 21 men, 4 women; 46.71 years $\pm 13.76 ; 72.49 \mathrm{~kg} \pm 9.73 ; 172.33 \mathrm{~cm} \pm 8.36$ ).

The subjects with UTA were wearing prostheses before being included (19 energy storage and return; 6 singleaxis feet prostheses; 25 patients had total surface bearing (TSB) prostheses). The post-amputation time prior to data collection was greater than or equal to 1 year $(10.17 \pm 9.29$ year, on average) for all individual with amputation. The persons were tested in their original prostheses and alignment. No statistically significant differences among groups were found for spatio-temporal parameters (table 1).

Joint internal moments are summarized in the table 2 . Figure 1 highlights the joint internal moments of the hip, knee, and ankle joints. The five graphs show the comparison between subjects with UTA and control individuals. For the controls, we checked that the curves were the same for gait cycles on the right and left sides. Therefore, we chose to illustrate the mean curves obtained for the right-side gait cycles.

Joint internal moments were statistically different among prosthetic limb, intact limb, and controls (table 2). We observed an increased hip extensor moment on the intact limb (vs. control: DM: .20 Nw/kg; CI95\% between .02 to .38; $\mathrm{p}=.02$ ) and on the prosthetic limb (vs. control: DM: $.22 \mathrm{Nw} / \mathrm{kg}$; CI95\% between .03 to $.40 ; \mathrm{p}=.014$ ) during the stance period.

The hip abductor moment was lower on the prosthetic limb compared to the intact limb (DM: .24Nw/kg; CI95\% between .05 to $.44 ; \mathrm{p}<.001$ ) and the control group (DM: $.25 \mathrm{Nw} / \mathrm{kg}$; CI95\% between .05 to .45; p=.007). The hip abductor moment on the intact limb was like the control group.

At the knee joint, the subjects with UTA walked with a reduced knee extensor and valgus moment on their prosthetic limb compared to the control group (extensor moment: DM: .22Nw/kg; CI95\% between .02 to .41; $\mathrm{p}=.02$; valgus: DM: .15Nw/kg; CI95\% between .02 to .27; $\mathrm{p}=.011$ ). No significant differences were observed between the knee moments on the intact limb and the knee moments of the control group during walking.

At the ankle joint, the statistical analysis showed that the people with UTA walked with a reduced plantarflexor moment during the stance period on the prosthetic limb (DM: .36Nw/kg; CI95\% between .17 to .55; p<.001) and on the intact limb (DM: .21Nw/kg; CI95\% between .008 to .39; $\mathrm{p}=.04$ ) compared to the persons without amputation.

\section{Discussion}

According to the results, asymmetric ankle, knee, and hip joint moments are presented in subjects with UTA.

The hip extensor moment was greater on the intact and prosthetic limbs compared to the control group. Furthermore, to protect the knee stability individuals with UTA develop compensatory motor strategies on their

Table 1: Comparison between groups: spatio-temporal data

\begin{tabular}{|l|c|c|c|c|c|}
\hline \multicolumn{1}{|c|}{ PARAMETERS } & Subjects with UTA & Control group & \multicolumn{3}{|c|}{ Subject with UTA vs. Control } \\
\cline { 3 - 5 } & & & DM & CI95\% & p \\
\hline Velocity $(\mathrm{m} / \mathrm{s})$ & $1.13( \pm .12)$ & $1.20( \pm .14)$ & .071 & $(-.03)-(.17)$ & .183 \\
\hline Cadence $($ steps $/ \mathrm{min})$ & $104.71( \pm 7.67)$ & $109.35( \pm 6.78)$ & 4.64 & $(-1.31)-(10.60)$ & .180 \\
\hline
\end{tabular}

Data are expressed in Mean $( \pm S D) * p<.001$ using Student's t-test. DM is difference of means. $95 \% \mathrm{Cl}$ is confidence interval of $95 \%$. GC is gait cycle

Table 2: Comparison between groups: kinetic data (joint internal moments)

\begin{tabular}{|c|c|c|c|c|c|c|c|c|c|c|c|c|}
\hline \multirow{2}{*}{$\begin{array}{l}\text { Joint moments } \\
\text { (peaks) } \mathrm{Nm} / \mathrm{kg}\end{array}$} & \multirow{2}{*}{$\begin{array}{l}\text { Prosthetic } \\
\operatorname{limb}\end{array}$} & \multirow{2}{*}{$\begin{array}{l}\text { Intact } \\
\text { limb }\end{array}$} & \multirow{2}{*}{$\begin{array}{l}\text { Control } \\
\text { group }\end{array}$} & \multicolumn{3}{|c|}{ Prosthetic vs. Intact } & \multicolumn{3}{|c|}{ Prosthetic vs. Control } & \multicolumn{3}{|c|}{ Intact vs. Control } \\
\hline & & & & DM & $\mathrm{Cl} 95 \%$ & $\mathbf{p}$ & DM & & $\mathbf{p}$ & DM & Cl95\% & $\mathbf{p}$ \\
\hline Hip extensor & $.43( \pm .29)$ & $.41( \pm .26)$ & $.21( \pm .14)$ & .01 & -.16 & .00 & .22 & to .40 & & .20 & 0.38 & $02 *$ \\
\hline & & & 7) & -.13 & & 79 & .06 & & & -.06 & & .00 \\
\hline & & & & .24 & to .44 & & .25 & & & .01 & & \\
\hline & & & & & & & & & & & & \\
\hline & & & & & & & & & & & & \\
\hline & $.28( \pm .19)$ & $.40( \pm .16)$ & $.43( \pm .13)$ & .11 & -.01 to .23 & .06 & .15 & & $.011^{*}$ & .04 & -.08 to .16 & 1.00 \\
\hline Ankle plantar flexor & $1.23( \pm .36)$ & $1.39( \pm .18)$ & $1.59( \pm .16)$ & .16 & -.02 to .35 & .10 & .36 & .17 to .55 & $<.001^{*}$ & .21 & .008 to .39 & $.04 *$ \\
\hline
\end{tabular}

Data are expressed in Mean $( \pm S D) * p<.001$ using one-way ANOVA (Bonferroni post hoc). DM is difference of means. $95 \% \mathrm{Cl}$ is confidence interval of $95 \%$. GC is gait cycle 
a)

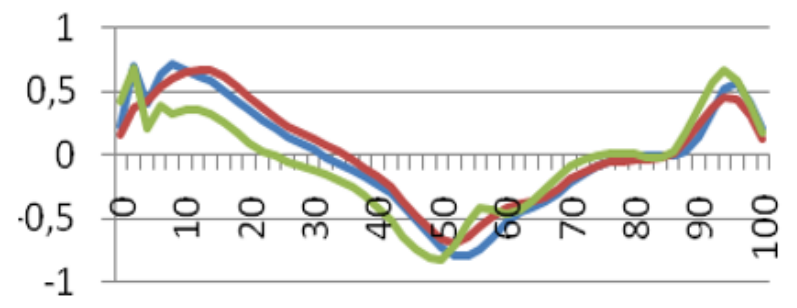

c)

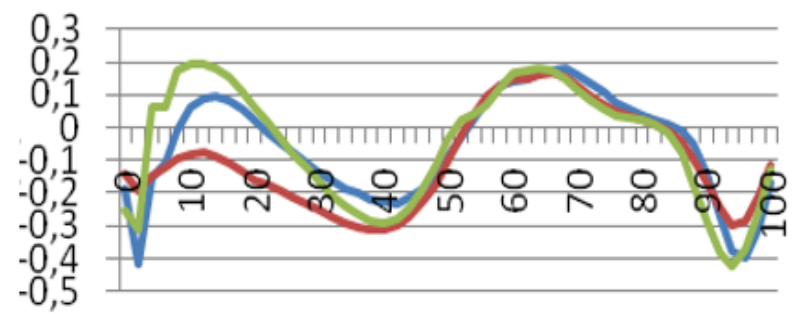

b)

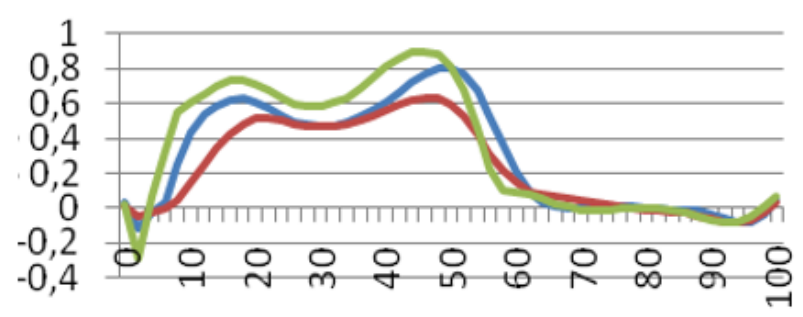

d)

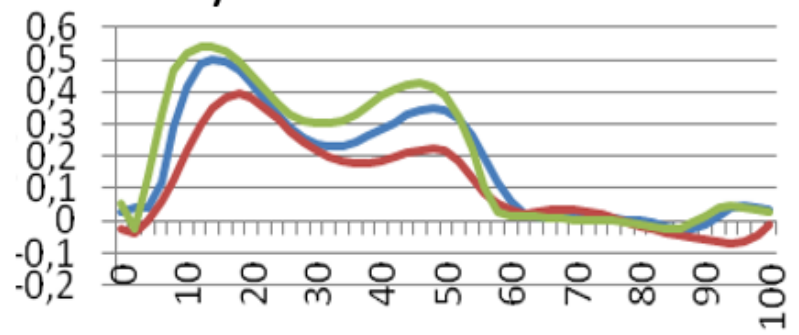

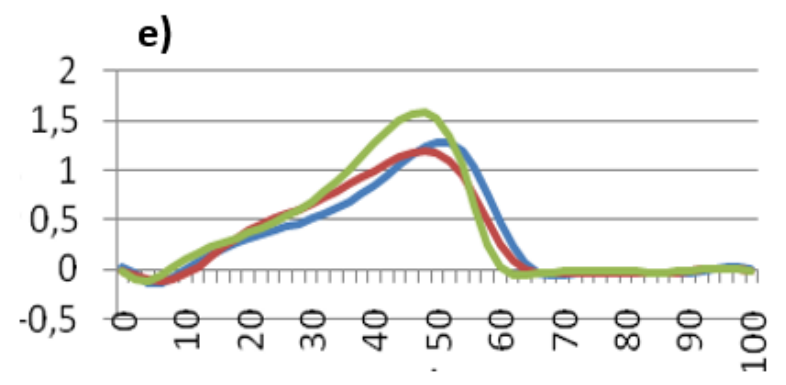

Figure 1: Joint moments plots during the gait cycle

a) Hip moment in the sagittal plane; b) Hip moment in the frontal plane; c) Knee moment in the sagittal plane; d) Knee moment in the frontal plane; e) Ankle moment in the sagittal plane. The red line is prosthetic limb, the blue line is intact limb, and the green line is control. $Y$ axis shows $\mathrm{Nm} / \mathrm{kg}$ and $\mathrm{X}$ axis shows the gait cycle $(0-100 \%)$

prosthetic limb through higher extensor forces at the hip joint. In addition, subjects with UTA overload their intact lower limb. Therefore, they develop compensatory motor strategies in the sagittal plane which are intended to unload and stabilize their prosthetic limb. These strategies, despite providing stability, may increase the risk of suffering degenerative joint disorders ${ }^{14,15}$. These findings are coherent with previous research ${ }^{16,17}$. In the frontal plane, the hip abductor moment on the intact limb was similar to the joint moment registered in controls. It is possible that the overload of the intact lower limb is mainly assumed by the hip extensor forces in comparison to abductor forces. On the other hand, the hip abductor moment was significantly decreased on the prosthetic limb which could indicate a medio-lateral instability in these people. Proper regulation of medio-lateral balance is associated with the hip work, particularly in the frontal plane where individuals with lower limb amputation are more unstable. In this sense, rehabilitation interventions should focus on hip muscle abductors to regulate mediolateral balance ${ }^{18}$.
At the knee joint, the internal moments of the intact limb were similar to those performed by non-amputated people. However, the knee extensor and valgus moments were lower in the prosthetic lower limb compared to the intact side. The joint moments registered for the amputated limb are likely to occur due to muscle weakness. Sibley et al. ${ }^{19}$, in a recent study, showed a decrease of the knee-extension moment in the prosthetic limb during gait in persons with UTA. This finding was accompanied by lower quadriceps muscle strength (60-76\%) and neural activation (32$44 \%$ ), slower contractile properties and altered muscle architecture in the amputated limb.

Considering the kinetic behavior of both lower limbs in the subjects with UTA and their relationship with the joint internal moments of non-amputated individuals, it is possible that the main strategy of motor compensation developed by people with UTA takes place in the intact lower limb at the hip joint in the sagittal plane, through a greater extensor moment, which is even higher than that developed by controls. These compensatory movements 
may contribute to poor ambulatory outcomes and the development of secondary pain conditions ${ }^{20}$ such as back pain, and intact limb joints pain ${ }^{21,22}$. However, the reduced joint internal moments observed on the prosthetic limb may contribute to increased instability. According to these findings, rehabilitation treatments following the amputation should include exercises for the strengthen of hip and knee muscles and for the balance training in the frontal plane in order to avoid joint instability, future degeneration and muscle dysfunction described in these subjects $^{19,23}$.

Another finding observed in individuals with UTA was the decrease of the ankle plantarflexor moment on the intact lower limb, compared to non-amputated persons. This kinetic pattern observed at the ankle joint could be explained by muscle fatigue. Yeung et al. ${ }^{24}$, observed in six people with UTA, that the intact limb ankle rocker progression and push-off were significantly reduced during walking. This may be due to the fatigue of the intact limb plantar flexors muscles in response to a decreased load on their prosthetic limb and the overload on the intact limb. The overload on the ankle joint at the intact limb may cause musculoskeletal alterations. In this sense, various studies have described findings of overload in the intact lower limb in amputee subjects.

This study has several limitations that should be mentioned. First, the placing of knee and ankle markers on the prosthesis at a location corresponding to the intact side might have affected the calculation of the joint centers. Additionally, the heterogeneous cohort and small sample size impeded the control of potential confounders, such as different times since amputation and since the current and first prosthesis prescription, differences in ages, differences in etiology of amputation and different prosthetic components.

\section{Conclusions}

Individuals with UTA walk with a greater hip extensor moment in both intact and prosthetic limbs compared to controls. The hip abductor moment and the knee extensor and valgus moments are reduced on the prosthetic limb. The ankle plantarflexor moment was decreased on the intact lower limb compared to controls, which may be indicative of fatigue.

\section{Conflicts of interest}

The authors have no conflicts of interest to report.

\section{Funding}

This research did not receive any specific grant from funding agencies in the public, commercial, or not-forprofit sectors.

\section{References}

1. Koelewijn AD, van den Bogert AJ. Joint contact forces can be reduced by improving joint moment symmetry in below-knee amputee gait simulations. Gait Posture. 2016; 49: 219-225.

2. Struyf PA1, van Heugten CM, Hitters MW, et al. The prevalence of osteoarthritis of the intact hip and knee among traumatic leg amputees. Arch Phys Med Rehabil. 2009; 90(3): 440-6.

3. Norvell DC, Czerniecki JM, Reiber GE, et al. The prevalence of knee pain and symptomatic knee osteoarthritis among veteran traumatic amputees and nonamputees. Arch Phys Med Rehabil. 2005; 86(3): 487-93.

4. Orekhov G, Robinson AM, Hazelwood SJ, et al. Knee joint biomechanics in transtibial amputees in gait, cycling, and elliptical training. PLoS One. 2019; 14(12): e0226060.

5. Beyaert C, Grumillier C, Martinet N, et al. Compensatory mechanism involving the knee joint of the intact limb during gait in unilateral below-knee amputees. Gait Posture. 2008; 28(2): 278-84.

6. Grumillier C, Martinet N, Paysant J, et al. Compensatory mechanisms involving the hip joint of the intact limb during gait in unilateral transtibial amputees. J Biomech. 2008; 41(14): 2926-31.

7. Molina Rueda F, Alguacil Diego IM, Molero Sánchez A, et al. Knee and hip internal moments and upper-body kinematics in the frontal plane in unilateral transtibial amputees. Gait Posture. 2013; 37(3): 436-9.

8. Molina-Rueda F, Alguacil-Diego IM, Cuesta-Gómez A, et al. Thorax, pelvis and hip pattern in the frontal plane during walking in unilateral transtibial amputees: biomechanical analysis. Braz J Phys Ther. 2014; 18(3): 252-8.

9. Krupenevich RL, Miller RH, Hendershot BD, et al. Knee adduction moment peak and impulse do not change during the first six months of walking with a prosthesis. Gait Posture. 2018; 63: 86-90.

10. Molina Carratala. Marcha humana: biomecánica, patología y tratamiento. Panamericina; 2020.

11. Vicon Plug-in Gait Product Guide-Foundation Notes Revision 2.0. Mar 2010.

12. Eng JJ, Winter DA. Kinetic analysis of the lower limbs during walking: what information can be gained from a three-dimensional model? J Biomech. 1995; 28(6): 753-8.

13. Batteni H, Olney SJ. Kinematic and kinetic variations of below-knee amputee gait. J Prosthet Orthot. 2002; 14: 2-10.

14. Royer TD, Wasilewski CA. Hip and knee frontal plane moments in persons with unilateral, trans-tibial amputation. Gait Posture. 2006; 23(3): 303-6.

15. Cho HM, Seo JW, Lee HJ, et al. Effect of surgical approach for contralateral side hip arthroplasty in below knee amputees: a retrospective cohort study. BMC Musculoskelet Disord. 2019; 20(1): 3.

16. Rusaw D, Ramstrand N. Motion-analysis studies of transtibial prosthesis users: a systematic review. Prosthetics and Orthotics International. 2011; 35(1): 8-19.

17. Soares AS, Yamaguti EY, Mochizuki L, et al. Biomechanical parameters of ait among transtibial amputees: a review. Sao Paulo Medical Journal. 2009; 127(5): 302-9.

18. Miller SE, Segal AD, Klute GK, et al. Hip recovery strategy used by below-knee amputees following mediolateral foot perturbations. J Biomech. 2018; 76: 61-67.

19. Sibley AR, Strike S, Moudy SC, et al. The effects of long-term muscle disuse on neuromuscular function in unilateral transtibial amputees. Exp Physiol. 2020; 105(3): 408-418.

20. Murray AM, Gaffney BM, Davidson BS, et al. Biomechanical 
compensations of the trunk and lower extremities during stepping tasks after unilateral transtibial amputation. Clin Biomech (Bristol, Avon). 2017; 49: 64-71.

21. Esfandiari E, Yavari A, Karimi A, et al. Long-term symptoms and function after war-related lower limb amputation: A national crosssectional study. Acta Orthop Traumatol Turc. 2018; 52(5): 348-351.

22. Allami M, Faraji E, Mohammadzadeh F, et al. Chronic musculoskeletal pain, phantom sensation, phantom and stump pain in veterans with unilateral below-knee amputation (published online ahead of print, 2019 Jul 4). Scand J Pain. 2019;/j/sjpain.ahead-of-print/ sjpain-2019-0045/sjpain-2019-0045.xml

23. Pröbsting E, Blumentritt S, Kannenberg A. Changes in the Locomotor System as a Consequence of Amputation of a Lower Limb. Z Orthop Unfall. 2017; 155(1): 77-91.

24. Yeung LF, Leung AK, Zhang $M$, et al. Long-distance walking effects on trans-tibial amputees compensatory gait patterns and implications on prosthetic designs and training. Gait Posture. 2012; 35(2): 32833. 